\title{
The Detection of Glycosphingolipids in Brain Tissue Sections by Imaging Mass Spectrometry Using Gold Nanoparticles
}

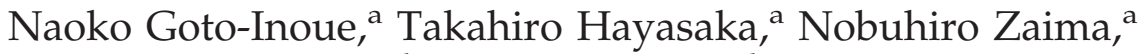 \\ Yukiyasu Kashiwagi, , Mari Yamamoto, ${ }^{\text {b }}$ Masami Nakamoto, ${ }^{\text {b }}$ and \\ Mitsutoshi Setou ${ }^{\mathrm{a}}$ \\ ${ }^{a}$ Department of Molecular Anatomy, Molecular Imaging Frontier Research Center, Hamamatsu University \\ School of Medicine, Shizuoka, Japan \\ ${ }^{\mathrm{b}}$ Osaka Municipal Technical Research Institute, Osaka, Japan
}

\begin{abstract}
Glycosphingolipids (GSLs) are amphiphilic molecules consisting of a hydrophilic carbohydrate chain and a hydrophobic ceramide moiety. They appear to be involved primarily in biological processes such as cell proliferation, differentiation, and signaling. To investigate the mechanism of brain function in more detail, a more highly sensitive method that would reveal the GSL distribution in the brain is required. In this report, we describe a simple and efficient method for mapping the distribution and localization of GSLs present in mouse brain sections using nanoparticle-assisted laser desorption/ionization imaging mass spectrometry (IMS). We have developed and tested gold nanoparticles (AuNPs) as a new matrix to maximize the detection of GSLs. A matrix of AuNPs modified with alkylamine was used to detect various GSLs, such as minor molecular species of sulfatides and gangliosides, in mouse brain sections; these GSLs were hardly detected using 2,5-dihydroxybenzoic acid (DHB), which is the conventional matrix for GSLs. We achieved approximately 20 times more sensitive detection of GSLs using AuNPs compared to a DHB matrix. We believe that our new approach using AuNPs in IMS could lead to a new strategy for analyzing basic biological mechanisms and several diseases through the distribution of minor GSLs. (J Am Soc Mass Spectrom 2010, 21, 1940-1943) (C) 2010 American Society for Mass Spectrometry
\end{abstract}

$\mathrm{T}$ The mouse brain contains a large amount of glycosphingolipids (GSLs). They are amphiphilic molecules consisting of a hydrophilic carbohydrate chain and a hydrophobic ceramide moiety. They appear to be involved primarily in biological processes such as cell proliferation, differentiation, and signaling $[1,2]$. Gangliosides are especially thought to play important roles in brain functions. To investigate the mechanism of brain function in more detail, a more highly sensitive method to reveal the GSL distribution in the brain is required. Imaging mass spectrometric (IMS) analyses of GSLs in the brain have been reported; however, assays using the conventional matrix could only indicate the distribution of major components of sulfatides and gangliosides [3]. Some reports have tried to detect other kinds of GSLs using an ionic liquid matrix [4] or their own newly developed matrix. Recently, Colsch and Woods succeeded in the visualization of minor gangliosides using their own matrix system [5]. To achieve high sensitivity, they applied their matrix in droplets by a chemical inkjet printer at low spatial resolution, although the most valuable point

Address reprint requests to Ms. N. Goto-Inoue, Department of Molecular Anatomy, Molecular Imaging Frontier Research Center, Hamamatsu University School of Medicine, 1-20-1 Handayama, Higashi-ku, Hamamatsu, Shizuoka 431-3192, Japan. E-mail: naoko.goto.inoue@gmail.com of IMS is the visualization of biomolecules with high spatial resolution.

IMS analyses using the conventional matrix, 2,5dihydroxybenzoic acid (DHB), have been developed to reveal the distribution of biomolecules such as phospholipids [6-8], proteins [9, 10], and GSLs [3, 5, 11]. The advantages of IMS are that it can visualize the distribution of biomolecules without derivatization and labeling and can identify the detailed structures of biomolecules on tissue sections.

Mass spectrometric (MS) analyses using nanoparticles (NPs) have become a popular technique due to the rapid development of nanomaterials. Mass spectrometry using NPs differs from spectrometry using organic matrices in several ways. For example, the mass spectra are simplified owing to a lack of chemical noise by the matrix [12]. The core metals gold [13], silver [14], and their mixture [15] have been used. Our research group applied NPs for IMS analyses using iron oxide and revealed the distributions of phosphatidylcholine and galactosylceramide in positive ion mode [16] and sulfatides in negative ion mode [17]. However, the detected ion intensity of sulfatides with NPs was much lower than that with DHB.

For this reason, we attempted to develop a new matrix system for IMS analyses to achieve higher spatial 
resolution as well as higher sensitivity. We developed and tested a new matrix solution for visualizing the distribution of GSLs in biological tissue sections using gold nanoparticles (AuNPs), which was modified with alkylamine. It is known that AuNPs can ionize GSLs with high sensitivity based on the results of the conventional MS analyses using NPs [13, 18]. In the present study, we could detect phosphatidylinositol (PI), sulfatides, and gangliosides species (GM3, GM2, GM1, GD1, and GD3) with high sensitivity in negative ion mode. Our application of AuNPs to the analysis of mouse brains achieved the desired visualization of minor components of GSLs. This is the first report of the visualization of minor GSLs by IMS analyses using AuNPs in biological sections.

\section{Experimental}

\section{Chemicals}

AuNPs were synthesized using the protocol described in the literature [19]. The AuNPs were prepared by controlled thermolysis of dimethylsulfide gold (I) chloride, $\mathrm{AuCl}\left(\mathrm{SMe}_{2}\right)$, as precursors in the presence of oleylamine. The mean diameter of AuNPs was $4.3 \pm 0.7$ $\mathrm{nm}$ determined by transmission electron microscopic images. Thermogravimetric analysis revealed that the metal content was $97 \%$. The DHB matrix was purchased from Bruker Daltonics (Bremen, Germany). Hexane, methanol, and ultra-pure water were purchased from Wako Pure Chemical (Osaka, Japan).

\section{Sample Preparation of Mouse Brain Sections in IMS Analyses}

Eight-wk-old male C57BL/6J mice were purchased from Japan SLC (Shizuoka, Japan). The mouse brain was frozen in liquid nitrogen, and serial sections were prepared to compare the difference in peaks detected by the spraying AuNPs and DHB as the matrix solution. The sections were sliced to a thickness of $10 \mu \mathrm{m}$ using a cryostat (CM 1950; Leica Microsystems, Wetzlar, Germany). Sections were thaw-mounted onto indiumtin-oxide (ITO)-coated glass slides (Bruker Daltonics). A thin AuNPs or DHB matrix layer was applied to the surface by an airbrush. Each tissue section was sprayed with $1000 \mu \mathrm{L}$ of AuNPs (50 mg/mL in hexane) or DHB matrix solution $(50 \mathrm{mg} / \mathrm{mL}$ in $70 \%$ methanol/water). IMS analyses were performed by raster-scanning in the $\mathrm{x}$-axis with a scan pitch of $200 \mu \mathrm{m}$ in the $\mathrm{y}$-axis.

\section{IMS Operating Conditions}

IMS analyses were performed using a MALDI-hybrid quadrupole TOF-type mass spectrometer (QSTAR Elite; AB SCIEX, Foster City, CA) equipped with an orthog-

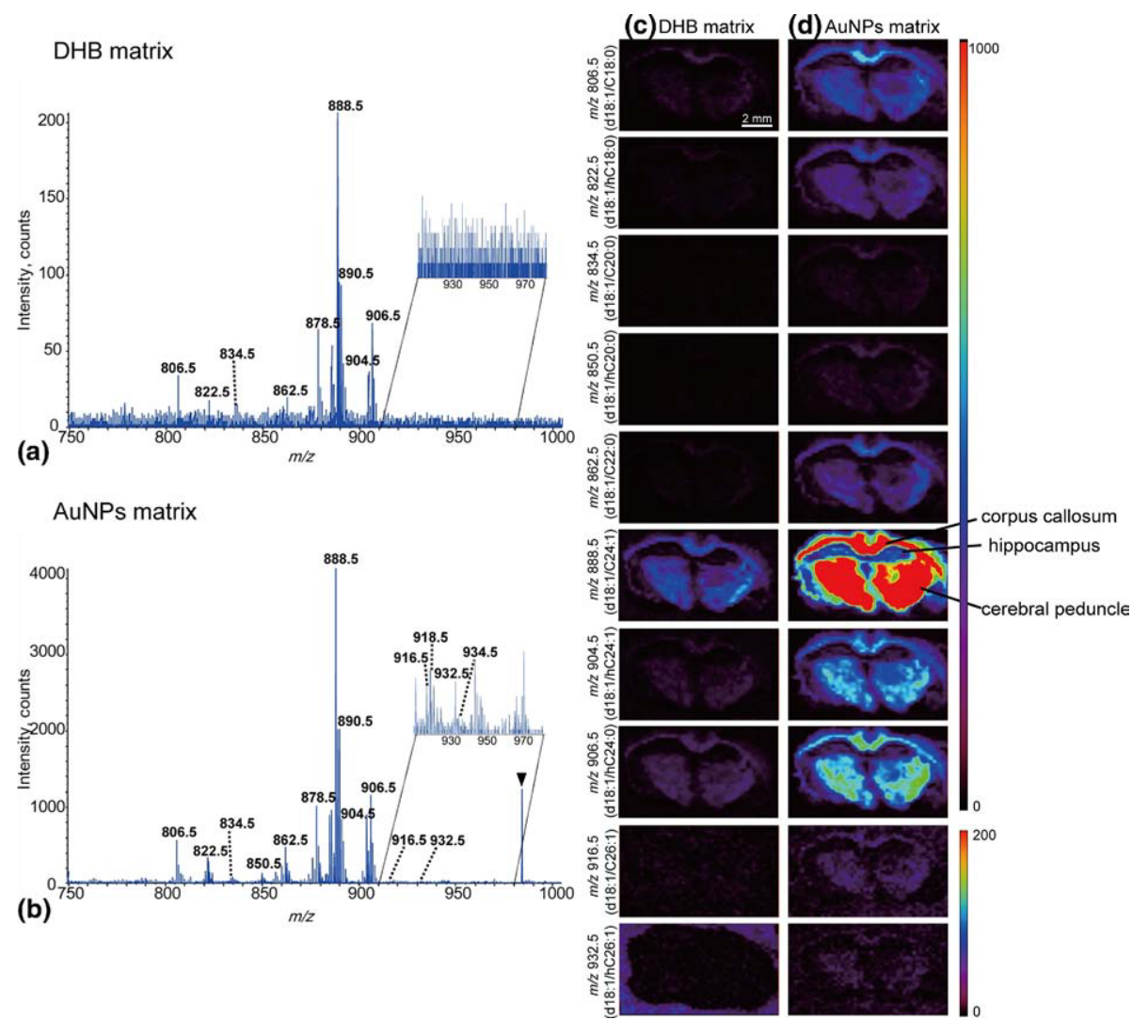

Figure 1. Negative-ion-mode MALDI imaging mass spectrum acquired from mouse brain tissue with DHB (a) and with AuNPs (b). The black triangle shows the peak derived from AuNPs. The ion images of 10 kinds of sulfatide molecular species with a DHB matrix (c) and AuNPs matrix (d). 
onal MALDI source and an Nd:YAG laser at a repetition rate of $1000 \mathrm{~Hz}$ with $60 \%$ laser power. Samples were analyzed in negative ion mode over the range of $\mathrm{m} / \mathrm{z}$ 500-2000. The ion images were constructed using BioMap software (Novartis, Basel, Switzerland).

\section{Results and Discussion}

\section{Comparison the Signal Intensity Between DHB and $A u N P S$}

Sulfatides have numerous molecular species owing to their fatty acid compositions. A previous study detected 10 kinds of sulfatides in the mouse brain with DHB matrix [20], though it was impossible to visualize all the molecular species by IMS analyses because of the low signals. Our AuNPs exhibited the high metal content as $97 \%$, and could be prepared the hexane solution without additives. Figure 1 presents the mass spectra directly acquired from brain sections with DHB (Figure 1a) and AuNPs (Figure 1b). We could detect $m / z 888.5$ as the major peak in both, however the signal intensity at $m / z 888.5$ with DHB was 206; on the contrary, that with AuNPs was 4000. We could identify the peak as sulfatide (d18:1/C24:1) according to MS/MS analysis and the previous report [17], and with 20 times higher sensitivity using AuNPs. We could also detect PIrelated ion such as at $m / z$ 885.5. The increased intensity of the sulfatide peak suggests that AuNPs constitute a suitable matrix for the visualization of sulfatides. Therefore, we investigated the detection of other sulfatides by IMS analyses. We succeeded in visualizing 14 kinds of sulfatide molecular species with AuNPs; m/z 806.5 (d18:1/C18:0), m/z 822.5 (d18:1/hC18:0), m/z 834.5 (d18: 1/C20:0), $m / z 850.5$ (d18:1/hC20:0), m/z 862.5 (d18:1/ C22:0), $m / z$ 878.5 (d18:1/hC22:0), $m / z 888.5$ (d18:1/C24: 1), $m / z \quad 890.5$ (d18:1/C24:0), m/z 904.5 (d18:1/hC24:1), m/z 906.5 (d18:1/hC24:0), m/z 916.5 (d18:1/C26:1), m/z 918.5 (d18:1/C26:0), $m / z 932.5$ (d18:1/hC26:1), and $m / z$ 934.5 (d18:1/hC26:0). On the contrary, we could not detect minor components of sulfatide $(\mathrm{m} / \mathrm{z} 850.5,916.5$, 918.5, 932.5, and 934.5) with DHB. We selected 10 sulfatides and compared their ion images by DHB and the AuNPs matrices (Figure $1 \mathrm{c}$ and $d$ ). We set the maximum intensity at 1000 and 200 to construct all ion images. With the DHB matrix, we could hardly describe ion images at $m / z 822.5,834.5,850.5,862.5,914.5,916.5$,

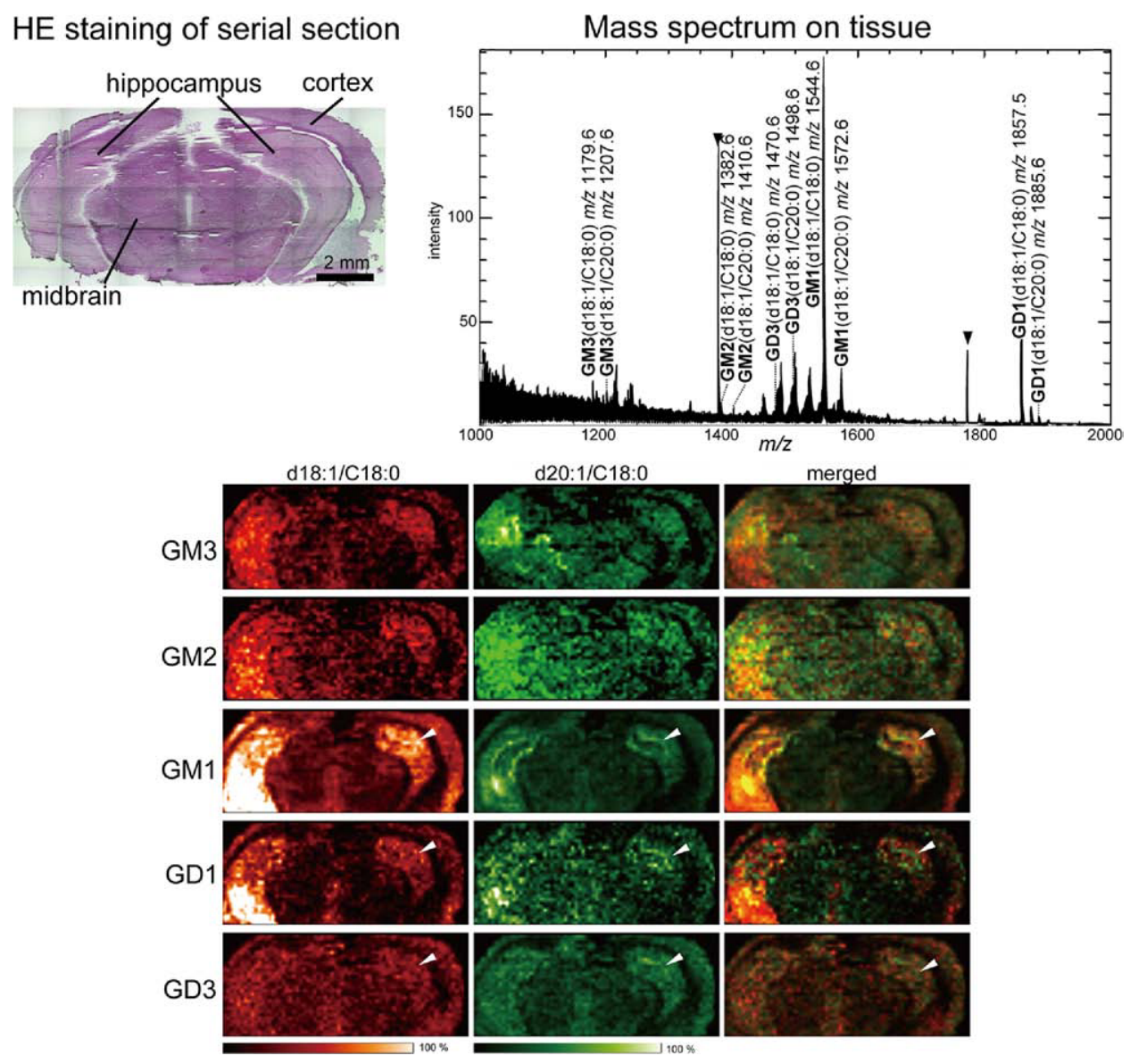

Figure 2. IMS analyses of gangliosides using AuNPs in negative-ion mode. The brain anatomy described in the HE staining result. Mass spectrum on tissue and different ion images for GM3, GM2, GM1, GD1, and GD3, depending on the sphingosine (d18:1 or d20:1), are shown. The black triangle on mass spectrum shows the peak derived from AuNPs. White arrows show the localization of molecular layer of the dentate gyrus in the mouse brain hippocampus. 
932.5, and 934.5. On the contrary, we could reliably obtain ion images of all of these ions with AuNPs, and confirmed that each intensity was approximately 20 times higher than with DHB. Therefore, we concluded that the use of AuNPs as a matrix in the negative ion mode improved the detection sensitivity for sulfatides.

\section{Ganglioside Mapping by IMS}

Next, we tried to show the application of AuNPs for visualization of gangliosides. Gangliosides consist of an oligosaccharide head group containing at least one sialic acid residue. This class of compounds presents a challenge for MS analysis because of the ready elimination of sialic acid [21]. As we conducted MS analysis of gangliosides using AuNPs, we could observe minor components of gangliosides (GM3, GM2, and GD3) as well as the major components (GM1 and GD1) (Figure 2). We showed brain anatomy on the HE stain image of the serial section. We also showed the mass spectrum on tissue. The signal intensities of peaks higher than $\mathrm{m} / \mathrm{z}$ 2000 was quite low with AuNPs; we could not get any signal relating to GT1 or GQ1, which have been detected by the other report [5]. All of these detected gangliosides have two kinds of molecular species owing to the difference of the sphingosine (d18:1 or $\mathrm{d} 20: 1)$. Both of GM3 (d18:1/C18:0 and d20:1/C18:0) and GM2 (d18:1/C18:0 and d20:1/C18:0) showed similar distributions, which are present mainly in the cortex and hippocampus formation. The results are consistent with the previous report [5]. The GM1 and GD1 (d18:1/ C18:0) appeared clearly in the cortex and hippocampus. On the other hand, the GM1 and GD1 (d20:1/C18:0) mappings were slightly different from results obtained for d18:1/C18:0. These results confirm recent studies, which show that GM1 and GD1 with d20:1 are mainly located in the molecular layer of the dentate gyrus in the mouse brain hippocampus [3]. We have to assign these molecules carefully because GD1 was eliminated of their sialic acids by laser irradiation [22]. The distributions of GD3 were also visualized though the ion intensity was quite low. We could realize a tendency for different distributions depending on the sphingoid base (d18:1 or d20:1) with GM1, GD1, and GD3. Further investigation to distinguish detailed mapping is needed.

\section{Conclusions}

Our study successfully demonstrated the visualization of GSLs in mouse brain samples by using AuNPs as a matrix for IMS analyses. The ion images from 14 kinds of sulfatides and 10 kinds of gangliosides were constructed. The use of AuNPs is expected to help elucidate several kinds of GSLs including minor molecular species.

\section{Acknowledgments}

The authors acknowledge support for this work by a Grant-in-Aid for SENTAN from the Japan Science and Technology Agency (to M.S.) and by a Grant-in-Aid for Young Scientists B (to N.G.-I.).

\section{References}

1. Hannun, Y. A.; Bell, R. M. Functions of Sphingolipids and Sphingolipid Breakdown Products in Cellular Regulation. Science 1989, 243(4890), 500-507.

2. Hakomori, S.; Handa, K.; Iwabuchi, K.; Yamamura, S.; Prinetti, A. New Insights in Glycosphingolipid Function: "Glycosignaling Domain," a Cell Surface Assembly of Glycosphingolipids with Signal Transducer Molecules, Involved in Cell Adhesion Coupled with Signaling. Glycobiology 1998, 8(10), xi-xix.

3. Sugiura, Y.; Shimma, S.; Konishi, Y.; Yamada, M. K.; Setou, M. Imaging Mass Spectrometry Technology and Application on Ganglioside Study Visualization of Age-Dependent Accumulation of C20-Ganglioside Molecular Species in the Mouse Hippocampus. PLoS One 2008, 3(9), e3232.

4. Chan, K.; Lanthier, P.; Liu, X.; Sandhu, J. K.; Stanimirovic, D.; Li, J. MALDI Mass Spectrometry Imaging of Gangliosides in Mouse Brain Using Ionic Liquid Matrix. Anal. Chim. Acta 2009, 639(1-2), 57-61.

5. Colsch, B.; Woods, A. S. Localization and Imaging of Sialylated Glycosphingolipids in Brain Tissue Sections by MALDI Mass Spectrometry. Glycobiology 2010, 20(6), 661-667.

6. Zaima, N.; Hayasaka, T.; Goto-Inoue, N.; Setou, M. Imaging of Metabolites by MALDI Mass Spectrometry. J. Oleo. Sci. 2009, 58(8), 415-419.

7. Shimma, S.; Sugiura, Y.; Hayasaka, T.; Zaima, N.; Matsumoto, M.; Setou, M. Mass Imaging and Identification of Biomolecules with MALDI-QIT-TOF-Based System. Anal. Chem. 2008, 80(3), 878-885.

8. Hayasaka, T.; Goto-Inoue, N.; Zaima, N.; Kimura, Y.; Setou, M. OrganSpecific Distributions of Lysophosphatidylcholine and Triacylglycerol in Mouse Embryo. Lipids 2009, 44(9), 837-848.

9. Andersson, M.; Groseclose, M. R.; Deutch, A. Y.; Caprioli, R. M. Imaging Mass Spectrometry of Proteins and Peptides: 3D Volume Reconstruction. Nat. Methods 2008, 5(1), 101-108.

10. Groseclose, M. R.; Andersson, M.; Hardesty, W. M.; Caprioli, R. M. Identification of Proteins Directly from Tissue: In Situ Tryptic Digestions Coupled with Imaging Mass Spectrometry. J. Mass Spectrom. 2007, 42(2), 254-262.

11. Shimma, S.; Sugiura, Y.; Hayasaka, T.; Hoshikawa, Y.; Noda, T.; Setou, M. MALDI-Based Imaging Mass Spectrometry Revealed Abnormal Distribution of Phospholipids in Colon Cancer Liver Metastasis. J. Chromatogr. B Analyt. Technol. Biomed. Life Sci. 2007, 855(1), 98-103.

12. Wen, X.; Dagan, S.; Wysocki, V. H. Small-Molecule Analysis with Silicon-Nanoparticle-Assisted Laser Desorption/Ionization Mass Spectrometry. Anal. Chem. 2007, 79(2), 434-444.

13. Wu, H. P.; Yu, C. J.; Lin, C. Y.; Lin, Y. H.; Tseng, W. L. Gold Nanoparticles as Assisted Matrices for the Detection of Biomolecules in a High-Salt Solution through Laser Desorption/Ionization Mass Spectrometry. J. Am. Soc. Mass Spectrom. 2009, 20(5), 875-882.

14. Chiu, T. C.; Chang, L. C.; Chiang, C. K.; Chang, H. T. Determining Estrogens Using Surface-Assisted Laser Desorption/Ionization Mass Spectrometry with Silver Nanoparticles as the Matrix. J. Am. Soc. Mass Spectrom. 2008, 19(9), 1343-1346.

15. Sherrod, S. D.; Diaz, A. J.; Russell, W. K.; Cremer, P. S.; Russell, D. H. Silver Nanoparticles as Selective Ionization Probes for Analysis of Olefins by Mass Spectrometry. Anal. Chem. 2008, 80(17), 6796-6799.

16. Taira, S.; Sugiura, Y.; Moritake, S.; Shimma, S.; Ichiyanagi, Y.; Setou, M. Nanoparticle-Assisted Laser Desorption/Ionization Based Mass Imaging with Cellular Resolution. Anal. Chem. 2008, 80(12), 4761-4766.

17. Ageta, H.; Asai, S.; Sugiura, Y.; Goto-Inoue, N.; Zaima, N.; Setou, M. Layer-Specific Sulfatide Localization in Rat Hippocampus Middle Molecular Layer is Revealed by Nanoparticle-Assisted Laser Desorption/ Ionization Imaging Mass Spectrometry. Med. Mol. Morphol. 2009, 42(1), $16-23$.

18. Jackson, S. N.; Ugarov, M.; Egan, T.; Post, J. D.; Langlais, D.; Albert Schultz, J.; Woods, A. S. MALDI-Ion Mobility-TOFMS Imaging of Lipids in Rat Brain Tissue. J. Mass Spectrom. 2007, 42(8), 1093-1098.

19. Yamamoto, M.; Kashiwagi, Y.; Nakamoto, M. Size-Controlled Synthesis of Gold Nanoparticles by Thermolysis of a Gold(I)-Sulfide Complex in the Presence of Alkylamines. Z Naturforsch. B 2009, 64(11-12), 13051311.

20. Jackson, S. N.; Wang, H. Y. J.; Woods, A. S. Direct Profiling of Lipid Distribution in Brain Tissue Using MALDI-TOFMS. Anal. Chem. 2005, 77(14), 4523-4527.

21. Harvey, D. J. Analysis of Carbohydrates and Glycoconjugates by Matrix-Assisted Laser Desorption/Ionization Mass Spectrometry: An Update Covering the Period 1999-2000. Mass Spectrom. Rev. 2006, 25(4), 595-662.

22. Costello, C. E.; Juhasz, P.; Perreault, H. New Mass Spectral Approaches to Ganglioside Structure Determinations. Prog. Brain Res. 1994, 101, 45-61. 